\title{
ON HOMOTOPY SPHERES BOUNDING HIGHLY CONNECTED MANIFOLDS
}

\author{
BY
}

DOUGLAS R. ANDERSON( $\left.{ }^{1}\right)$

Let $\alpha \in \pi_{n-1}\left(S O_{m}\right), \beta \in \pi_{m-1}\left(S O_{n}\right)$ and consider the manifold $N_{\alpha, \beta}$ obtained by plumbing together the total spaces $E_{\alpha}$ and $E_{\beta}$ of the $D^{m}$ (respectively $D^{n}$ ) bundle over $S^{n}$ (respectively $S^{m}$ ) with characteristic classes $\alpha$ (respectively $\beta$ ). In [10] Milnor shows that $\partial N_{\alpha, \beta}$ is almost always a homotopy sphere. In particular, if $m=n$ and $\alpha, \beta \in \operatorname{Im}\left(\pi_{n-1}\left(S O_{n-1}\right) \rightarrow \pi_{n-1}\left(S O_{n}\right)\right)$, then $\partial N_{\alpha, \beta}$ is a homotopy sphere of dimension $(2 n-1)$. Let $\Lambda_{2 n-1}$ be the set of all such spheres. It is the object of this note to investigate these spheres.

It is shown in [12] (and [8] by quite different methods) that $\Lambda_{2 n-1}$ is a subgroup of $\Theta_{2 n-1}$, the group of homotopy $(2 n-1)$ spheres, whose elements represent obstructions to smoothing $(n-1)$ connected PL manifolds of dimension $2 n$ where $n$ skeleton can be smoothed. It is of interest, then, to know what the subgroup $\Lambda_{2 n-1}$ is. In this connection we prove

THEOREM 1. Let $n=4 m$ with $m>2$ and $\tau_{m}=\sigma_{2 m}$ /g.c.d. $\left[\sigma_{m}^{2}, \sigma_{2 m}\right]$ where

$$
\sigma_{k}=2^{2 k}\left(2^{2 k-1}-1\right) B_{k} a_{k} j_{k} / 2 k,
$$

$B_{k}$ is the kth Bernoulli number, $a_{k}$ is 1 or 2 depending on whether $k$ is even or odd, and $j_{k}$ is the order of the image of the stable J homomorphism, $J: \pi_{4 k-1}(S O) \rightarrow \pi_{4 k-1}^{S}$. Then $\Lambda_{2 n-1}$ is a cyclic group of order dividing $j_{m}^{2} \cdot \tau_{m}$. Furthermore $\Lambda_{8 m-1}$ contains a subgroup of $\Theta_{8 m-1}(\partial \pi)$ of order $\tau_{m}$.

Let $P^{8 m}$ be the $(4 m-1)$ connected $\pi$ manifold of index 8 with $\partial P$ a generator of $\Theta_{8 m-1}(\partial \pi)$. Let $M^{8 m}$ be the closed PL manifold obtained from $P$ by attaching a cone over the boundary. As an application of Theorem 1 we prove

THEOREM 2. $M^{8 m}$ does not have the homotopy type of a closed differentiable manifold.

Since the manifold $M$ of Theorem 2 satisfies Poincare duality and the trivial bundle over $M$ is reducible, the Browder-Novikov Theorem on the homotopy type of manifolds [3], [11] gives the

COROLlaRY. There is no reducible vector bundle over $M$ whose dual Pontrjagin classes satisfy the index theorem.

Received by the editors February 2, 1968 and, in revised form, May 28, 1968.

(1) Partially supported by the National Science Foundation under grant no. NSF GP 6961. 
1. Proofs of Theorems 1 and 2. Theorems 1 and 2 are both consequences of Lemma 1.5 below. We state the lemmas needed to prove 1.5 first, but defer their proofs to $\$ 2$. We assume the reader is familiar with the theory in [12].

Let $X$ be an $(n-1)$ connected space satisfying Poincaré duality for the dimension $2 n$. Let $\mu_{X} \in H_{2 n}(X)$ be the orientation class.

LEMMA 1.1. Let $\zeta^{k}$ be a reducible vector bundle over $X, k \gg 2 n$. Then there are oriented $(n-1)$ connected smooth handlebodies $N, P$ with $P$ parallelizable and maps $f, \tilde{f}$ such that

(i) $\partial N=-\partial P$ is a homotopy sphere.

(ii) $f: N \cup P \rightarrow X$ and $f ! \zeta=\nu$ is the stable normal bundle of $N \cup P$ where $N \cup P$ is the oriented smooth manifold obtained from the disjoint union of $N$ and $P$ by gluing along the boundaries via the identity map.

(iii) $\bar{f}: \hat{N} \rightarrow X$ is a homotopy equivalence where $\hat{N}$, the closure of $N$, is obtained from $N$ by attaching the cone over the boundary.

(iv) The diagram

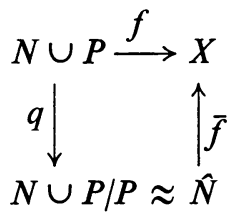

is homotopy commutative.

Proof. The proof is given in $\S 2$.

If $n$ is even, $H J \oplus S: \pi_{n-1}\left(S O_{n}\right) \rightarrow Z \oplus \pi_{n-1}(S O)$ is a monomorphism where $H$ is the Hopf invariant, $J$ the $J$ homomorphism, and $S$ the suspension. Hence if $(H ;\langle,\rangle ; \alpha)$ is an $n$-space, $\alpha$ is completely determined by $H J \alpha$ and $S \alpha$. By recalling that $H J \alpha(x)=\langle x, x\rangle$ and $S \alpha$ is a homomorphism, we will consider an $n$-space ( $n$ even) to be a triple $(H ;\langle,\rangle ; \beta)$ where $H$ is a free abelian group, $\langle$,$\rangle is a non-$ singular bilinear form on $H$; and $\beta: H \rightarrow \pi_{n-1}(S O)$ is a homomorphism.

If $\zeta^{k}$ is any stable bundle over $X$, we may define a map

$$
\beta_{\zeta}: H_{n}(X) \rightarrow \pi_{n-1}(S O)
$$

by observing that every element $x \in H_{n}(X)$ is spherical. Thus there is a map $f_{x}: S^{n} \rightarrow X$ representing $x$. Let $\beta_{\zeta}(x)$ be the characteristic class of $f_{x}^{!} \zeta$. Clearly $\beta_{\zeta}$ is a homomorphism.

LeMmA 1.2. Let $n$ be even. Let $X, \zeta^{k}$, and $N$ be as in 1.1. Then $N$ is the handlebody corresponding to the $n$-space $\left(H_{n}(X) ;\langle\rangle ;,-\beta_{\zeta}\right)$. Thus $N$ is determined completely by $X$ and $\zeta$.

Proof. See $\S 2$.

Let $n=4 m$ and $\lambda, \mu$ be integers. Then $N_{\lambda, \mu}$ will denote the handlebody obtained by plumbing together the total spaces of the $D^{n}$ bundles over $S^{n}$ with characteristic 
classes $\lambda^{\prime}, \mu^{\prime} \in \pi_{n-1}\left(S O_{n}\right)$ satisfying $(H J \oplus S)\left(\lambda^{\prime}\right)=(0, \lambda)$ and $(H J \oplus S)\left(\mu^{\prime}\right)=(0, \mu)$. Since $H J\left(\lambda^{\prime}\right)=0, \lambda^{\prime} \in \operatorname{Im}\left(\pi_{n-1}\left(S O_{n-1}\right) \rightarrow \pi_{n-1}(S O)\right)$ and $\partial N_{\lambda, \mu}$ is a homotopy sphere (see [10]). We note for future reference that the $n$ space corresponding to $N_{\lambda, \mu}$ has $H=Z+Z$ with generators $e_{1}$ and $e_{2}$. Relative to this basis, $\langle$,$\rangle has$ matrix

$$
\left(\begin{array}{ll}
0 & 1 \\
1 & 0
\end{array}\right)
$$

Finally $S \alpha\left(e_{1}\right)=\lambda, S \alpha\left(e_{2}\right)=\mu$.

The order of the image of $J: \pi_{4 m-1}(S O) \rightarrow \pi_{4 m-1}^{S}$ will be denoted by $j_{m}$.

LemMA 1.3. Let $n=4 m$ and $N_{i}^{2 n}, i=1,2$, be $(n-1)$ connected handlebodies with boundaries homotopy spheres. If the closures $\hat{N}_{i}$ of $N_{i}$ have the same homotopy type, then there is an integer $\lambda$ such that $\partial N_{1}=\partial N_{2} \# \partial N_{\lambda, j_{m}}$.

Proof. The proof is given in $\S 2$.

LeMmA 1.4. $\lambda \partial N_{1, \mu}=\mu \partial N_{\lambda, 1}$ and $\partial N_{\lambda, \mu}=\lambda \mu \cdot \partial N_{1,1}$.

Proof. See $\S 2$.

Theorems 1 and 2 are both consequences of the following key:

LemMA 1.5. Let $n=4 m(m>2)$. Then $\partial N_{j_{m}, j_{m}}$ is an element of order $\tau_{m}$.

Proof. Let $\zeta_{r}$ be the $l$-plane bundle over $S^{n}, l \gg 4 m$, with characteristic class $r j_{m}$, $r=0, \pm 1, \pm 2, \ldots$ Then $\zeta_{r}$ is fiber homotopically trivial for any $r$. Let $X=S_{1}^{n} \times S_{2}^{n}$ and $\pi_{i}: X \rightarrow S_{i}^{n}, i=1,2$, be the projections. Then $\xi=\pi_{1}^{!}\left(\zeta_{-1}\right) \oplus \pi_{2}^{!}\left(\zeta_{-t}\right)$ is a fiber homotopically trivial bundle over $X$. Thus $\xi$ has the same fiber homotopy type as the stable normal bundle of $X$. Hence $\xi$ is reducible. By 1.1 and 1.2 , then, there is a unique handlebody $N$ with $\partial N \in \Theta_{2 n-1}(\partial \pi)$ and a homotopy equivalence

$$
\bar{f}: N \cup C(\partial N) \rightarrow X
$$

such that $\bar{f}^{\prime} \xi \mid N$ is the stable normal bundle of $N$. In fact $N$ is the handlebody corresponding to the $n$-space $\left(H_{n}(X),\langle\rangle,,-\beta_{\xi}\right)$. Let $\dot{e}_{i} \in H_{n}(X)$ correspond to the inclusion $S_{i}^{n} \rightarrow S_{1}^{n} \times S_{2}^{n}, i=1,2$. Then $e_{1}, e_{2}$ are generators of $H_{n}(X)=Z \oplus Z$ satisfying $e_{i} e_{j}=1-\delta_{i j}$ and $-\beta_{\xi}\left(e_{1}\right)=j_{m},-\beta_{\xi}\left(e_{2}\right)=t j_{m}$. Since this is exactly the $n$-space corresponding to $N_{j_{m}, t j_{m}}$ it follows that $\partial N_{j_{m}, t j_{m}} \in \Theta_{2 n-1}(\partial \pi)$ for all $t$.

In order to compute the order of $\partial N_{j_{m}, t j_{m}}$ in $\Theta_{2 n-1}(\partial \pi)$, we compute the index $\tau(P)$ of the $\pi$-manifold $P$ of 1.1 . But, in the notation of 1.1 , since $f^{\prime} \xi$ is the stable normal bundle of $P \cup N$,

$$
\begin{array}{r}
\tau(P)=\tau(P)+\tau(N)=\tau(P \cup N)=\left\langle L_{2 m}\left(\bar{p}_{1}\left(f^{\prime} \xi\right), \ldots, \bar{p}_{2 m}\left(f^{\prime} \xi\right)\right), \mu_{P \cup N}\right\rangle \\
=\left\langle L_{2 m}\left(\bar{p}_{1}(\xi), \ldots, \bar{p}_{2 m}(\xi)\right), \mu_{X}\right\rangle .
\end{array}
$$

(The last equality follows since $f_{*} \mu_{P \cup N}=\mu_{X}$.)

Simple computations using the Whitney sum formula show that

$$
\bar{p}_{m}(\xi)=-\left[p_{m}\left(\zeta_{-1}\right) \times 1+1 \times p_{m}\left(\zeta_{-t}\right)\right], \quad \bar{p}_{2 m}(\xi)=p_{m}\left(\zeta_{-1}\right) \times p_{m}\left(\zeta_{-t}\right) .
$$


Thus $2 \bar{p}_{2 m}(\xi)=\left(\bar{p}_{m}(\xi)\right)^{2}$. In the case that the only nonzero Pontrjagin classes are in dimensions $m$ and $2 m, L_{2 m}=s_{2 m} p_{2 m}+\frac{1}{2}\left(s_{m}^{2}-s_{2 m}\right) p_{m}^{2}$ where $s_{i}=2^{2 i}\left(2^{2 i-1}-1\right) B_{i} /(2 i)$ !. Thus $L_{2 m}\left(\bar{p}_{m}(\xi), \bar{p}_{2 m}(\xi)\right)=s_{m}^{2} p_{2 m}(\xi)=s_{m}^{2} p_{m}\left(\zeta_{-1}\right) \times p_{m}\left(\zeta_{-t}\right)$. By a formula due to Kervaire $p_{m}\left(\zeta_{r}\right)=a_{m}(2 m-1) ! r j_{m} e$ for an appropriate generator $e \in H^{n}\left(S^{n}\right)$. Since we may orient $X$ in any desired fashion we may suppose that $\mu_{X}=e \times e$. Substituting for $s_{m}, p_{m}\left(\zeta_{-1}\right)$, and $p_{m}\left(\zeta_{-t}\right)$ and combining the formulae above then gives

$$
\tau(P)=\sigma_{m}^{2} t .
$$

By [7, Theorem 7.5], $\partial N_{j_{m}, t j_{m}}=\partial P=S^{8 m-1}$ if and only if $\tau(P) \equiv 0\left(\bmod \sigma_{2 m}\right)$. Thus $\partial N_{j_{m}, t j_{m}}=S^{8 m-1}$ if and only if $\sigma_{2 m} \mid \sigma_{m}^{2} t$, i.e. if and only if $\sigma_{2 m} /$ g.c.d. $\left[\sigma_{m}^{2}, \sigma_{2 m}\right]$ divides $t$. Since $\partial N_{j_{m}, t j_{m}}=t \partial N_{j_{m}, j_{m}}$ (cf. 1.4) the lemma follows.

We now prove Theorems 1 and 2.

Proof of Theorem 1. It follows directly from [12, Theorem 2 (1)] that $\Lambda_{2 n-1}$ is a cyclic group generated by $\partial N_{1,1}$. An argument similar to 1.4 however shows that $\partial N_{j_{m}, j_{m}}=j_{m}^{2} \partial N_{1,1}$. Thus Theorem 1 follows from 1.5.

Proof of Theorem 2. Suppose that $M$ has the homotopy type of a closed differentiable manifold $M^{\prime}$. Then $M^{\prime}$ is the closure of a $(4 m-1)$ connected handlebody $N^{\prime}$ with $\partial N^{\prime}=S^{2 n-1}$ and by $1.3 \partial P=S^{2 n-1} \# \partial N_{\lambda, j_{m}}=\partial N_{\lambda, j_{m}}$. Since $\partial P$ generates $\Theta_{8 m-1}(\partial \pi)$, the theorem follows by proving that $\partial N_{\lambda, j_{m}}$ cannot be a generator of $\Theta_{8 m-1}(\partial \pi)$.

To see the last statement we recall that since $\partial N_{j_{m}, j_{m}}=j_{m} \partial N_{1, j_{m}}$ by 1.4 and the order of $\partial N_{j_{m}, j_{m}}=\tau_{m}$, the order of $\partial N_{1, j_{m}}$ divides $j_{m} \tau_{m}$. Thus the power of 2 in the order of $\partial N_{1, j_{m}}$ is less than or equal to the power of 2 in $j_{m} \tau_{m}$. But an easy computation shows that $\tau_{m}$ is odd and it is well known that the power of 2 in $j_{m}$ is less than the power of 2 in $16 m$, at least for $m \geqq 3$. Thus the power of 2 in the order of $\partial N_{1, j_{m}}$ is less than that in $16 m$. By Kervaire-Milnor [7], the order of $\Theta_{8 m-1}(\partial \pi)$ is $\sigma_{2 m} / 8$. An easy computation now shows that the power of 2 in $16 \mathrm{~m}$ is less than that in $\sigma_{2 m} / 8$. Thus $\partial N_{1, j_{m}}$ does not generate $\Theta_{8 m-1}(\partial \pi)$ and $\partial N_{\lambda, j_{m}}=\lambda \partial N_{1, j_{m}}$ cannot generate $\Theta_{8 m-1}(\partial \pi)$.

The author would like to thank the referee for suggesting the argument above which greatly simplifies the original proof.

2. Proof of Lemmas 1.1-1.4. In this section we prove the lemmas used in the proofs of Theorems 1 and 2 . The proofs rely heavily on the classification theory of [12] and we shall assume the reader's familiarity with the theory and notation of that paper. In particular we shall use $n$-spaces and the computation of the Grothendieck group of $n$-spaces freely.

Proof of 1.1. Let $g: S^{2 n+k} \rightarrow T(\zeta)$ be such that the image of [g] under the Hurewicz homomorphism is the generator $\phi\left(\mu_{X}\right) \in H_{2 n+k}(T(\zeta))$ where $\phi$ is the Thom isomorphism. By the usual methods of transverse regularity and surgery we may assume $M=g^{-1}(X)$ is $(n-1)$ connected. We shall decompose $M$ in the desired fashion by showing that the $n$-space $\left(H_{n}(M) ;\langle,\rangle ; \alpha\right)$ corresponding to $M$ may be decomposed as the direct sum of $n$-spaces. 
Let $f=g \mid M$ and $\mu_{M} \in H_{2 n}(M)$ be the orientation class satisfying $f_{*} \mu_{M}=\mu_{X}$. Let

$$
\begin{aligned}
& H^{\prime}=\operatorname{ker}\left(f_{*}: H_{n}(M) \rightarrow H_{n}(X)\right), \\
& H^{\prime \prime}=\operatorname{im}\left(\left(\cap \mu_{m}\right) f^{*}: H^{n}(X) \rightarrow H_{n}(M)\right) .
\end{aligned}
$$

Then it is well known that $H_{n}(M) \approx H^{\prime} \oplus H^{\prime \prime}$ and that this splitting extends to the intersection pairing; that is $\langle x, y\rangle=0$ for any $x \in H^{\prime}, y \in H^{\prime \prime}$. Thus if $z=x+y$ is an element of $H_{n}(M)$ with $x \in H^{\prime}, y \in H^{\prime \prime}, \alpha(z)=\alpha(x+y)=\alpha(x)+\alpha(y)+\langle x, y\rangle \partial \iota_{n}=$ $\alpha(x)+\alpha(y)$. Hence $\left(H_{n}(M) ;\langle,\rangle ; \alpha\right)=\left(H^{\prime} ;\langle,\rangle^{\prime} ; \alpha^{\prime}\right) \oplus\left(H^{\prime \prime} ;\langle,\rangle^{\prime \prime} ; \alpha^{\prime \prime}\right)$ where $\langle,\rangle^{\prime}=\langle\rangle,\left|H^{\prime} ; \alpha^{\prime}=\alpha\right| H^{\prime} ;\langle,\rangle^{\prime \prime}=\langle\rangle,\left|H^{\prime \prime} ; \alpha^{\prime \prime}=\alpha\right| H^{\prime \prime}$. Since $\langle$,$\rangle is non-$ singular, so are $\langle,\rangle^{\prime}$ and $\langle,\rangle^{\prime \prime}$ and the $n$-space for $M$ is decomposed as stated.

Let $P$ and $N$ be the handlebodies corresponding to

$$
\left(H^{\prime} ;\langle,\rangle^{\prime} ; \alpha^{\prime}\right) \text { and }\left(H^{\prime \prime} ;\langle,\rangle^{\prime \prime} ; \alpha^{\prime \prime}\right)
$$

respectively. It follows immediately from the construction that $\partial P$ and $\partial N$ are homotopy spheres and that $M^{2 n}-\operatorname{int}\left(D^{2 n}\right)$ is diffeomorphic to the boundary connected sum of $P$ and $N$. Thus $S^{2 n-1}=\partial P \# \partial N, \partial P=-\partial N$ and $M$ is almost diffeomorphic to $P \cup N$. In the sequel, we identify $M$ with $P \cup N$ under this almost diffeomorphism. Since almost diffeomorphic manifolds have the same stable normal bundle, $f \zeta \zeta$ is the stable normal bundle of $P \cup N$.

The handlebody $P$ is a $\pi$-manifold for if $h: S^{n} \rightarrow M$ is an embedding representing any element $x \in H^{\prime}$, since $f_{*} x=0, f^{!} \zeta\left|h\left(S^{n}\right)=\nu_{M}\right| S^{n}$ is trivial where $\nu_{M}$ denotes the stable normal bundle of $M$. Thus the normal bundle $\nu\left(h\left(S^{n}\right), M\right)$ of $h\left(S^{n}\right)$ in $M$ is stably trivial since $\alpha(x)$ is the characteristic of $\nu\left(h\left(S^{n}\right), M\right)$; this means that $S \alpha(x)=0$ for any $x \in H^{\prime}$. Hence $P$ is a $\pi$-manifold.

It follows that $f \mid P$ is null homotopic and that there is a map $\bar{f}: \hat{N} \rightarrow X$ such that the diagram in (iv) is homotopy commutative.

To see (iii) note that since $f_{*}: H_{n}(N \cup P) \rightarrow H_{n}(X)$ is onto, so is $\bar{f}_{*}$. On the other hand, since $H_{n}(P)=H^{\prime}=\operatorname{ker} f_{*}=\operatorname{ker} \bar{f}_{*} q_{*}: H_{n}(N \cup P) \rightarrow H_{n}(X)$ and $H_{n}(P)=$ $\operatorname{ker} q_{*}: H_{n}(N \cup P) \rightarrow H_{n}(N \cup P, P) \approx H_{n}(N \cup P / P), \bar{f}_{*}$ is a monomorphism in dimension $n$. Finally since $f$ and $q$ have degree 1 , so does $\bar{f}$. Thus $\bar{f}_{*}: H_{*}(\hat{N}) \rightarrow H_{*}(X)$ is an isomorphism and (iii) follows.

Proof of 1.2. It is easy to see that the map $\bar{f}$ of 1.1 induces an isomorphism of the $n$-space corresponding to $N$ with $\left(H_{n}(X) ;\langle\rangle ;,-\beta_{\zeta}\right)$.

Proof of 1.3. The proof is divided into two cases.

Case 1. The index $\tau\left(\hat{N}_{1}\right)=0=\tau\left(\hat{N}_{2}\right)$. In this case since the quadratic form $\langle x, x\rangle$, $x \in H_{n}\left(N_{1}\right)=H_{n}\left(\hat{N}_{1}\right)$, assumes only even values and is unimodular, there is a sympletic basis $b_{1}, \ldots, b_{s}, c_{1}, \ldots, c_{s}$ for $H_{n}\left(\hat{N}_{1}\right)$. Thus $b_{i} b_{j}=0=c_{i} c_{j}$ and $b_{i} c_{j}=\delta_{i j}$.

Let $f: \hat{N}_{1} \rightarrow \hat{N}_{2}$ be a homotopy equivalence. Then $f_{*}: H_{*}\left(\hat{N}_{1}\right) \rightarrow H_{*}\left(\hat{N}_{2}\right)$ preserves intersections and the map $J \alpha$; see [12, Lemma 8]. Then $b_{i}^{\prime}=f_{*} b_{i}, c_{i}^{\prime}=f_{*} c_{i}$, $i=1, \ldots, s$, is a sympletic basis for $H_{n}\left(\hat{N}_{2}\right)$ and $J \alpha_{1}\left(b_{i}\right)=J \alpha_{2}\left(b_{i}^{\prime}\right)$. Thus $\alpha_{1}\left(b_{i}\right)-\alpha_{2}\left(b_{i}^{\prime}\right)$ $\in \operatorname{ker}\left(J: \pi_{n-1}\left(S O_{n}\right) \rightarrow \pi_{2 n-1}\left(S^{n}\right)\right)$ and since 


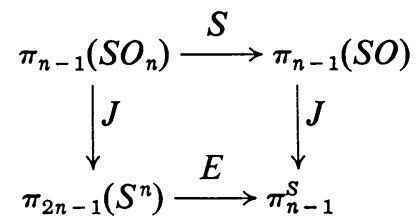

commutes up to sign, $J S\left(\alpha_{1}\left(b_{i}\right)-\alpha_{2}\left(b_{i}^{\prime}\right)\right)=0$. Hence, since $n=4 m, S \alpha_{1}\left(b_{i}\right)=S \alpha_{2}\left(b_{i}^{\prime}\right)+$ $\beta_{i} j_{m}$ for some integer $\beta_{i}$ where we have identified $j_{m}$ with a generator of the kernel of the stable $J$ homomorphism. Similarly $S \alpha_{1}\left(c_{i}\right)=S \alpha_{2}\left(c_{i}^{\prime}\right)+\gamma_{i} j_{m}$ for some integer $\gamma_{i}$.

Following Wall, let $\chi_{k} \in H_{n}\left(\hat{N}_{k}\right), k=1,2$, be such that $\left\langle\chi_{k}, x\right\rangle=S \alpha(x)$ for any $x \in H_{n}\left(\hat{N}_{k}\right)$. Since $b_{1}, \ldots, b_{s}, c_{1}, \ldots, c_{s}$ is a sympletic basis $\chi_{1}=\sum_{i=1}^{s} S \alpha_{1}\left(c_{i}\right) b_{i}+$ $\sum_{i=1}^{s} S \alpha_{1}\left(b_{i}\right) c_{i}$. Similarly $\chi_{2}=\sum_{i=1}^{s} S \alpha_{2}\left(c_{i}^{\prime}\right) b_{i}^{\prime}+\sum_{i=1}^{s} S \alpha_{2}\left(b_{i}^{\prime}\right) c_{i}^{\prime}$. Let

$$
\lambda=\sum_{i=1}^{s}\left(\gamma_{i} S \alpha_{2}\left(b_{i}^{\prime}\right)+\beta_{i} S \alpha_{2}\left(c_{i}^{\prime}\right)+\beta_{i} \gamma_{i} j_{m}\right)
$$

and consider $N_{1}^{\prime}=N_{1} \boxminus\left(S^{n} \times S^{n}-\dot{D}^{2 n}\right)$ and $N_{2}^{\prime}=N_{2} \sharp N_{\lambda, j_{m}}$ where 4 denotes boundary connected sum. We shall show that $N_{1}^{\prime}$ and $N_{2}^{\prime}$ determine the same element in the Grothendieck group of $n$-spaces.

By [12, Theorem 2, (1)], the element of $\mathscr{G}_{n}$ corresponding to any $(n-1)$ connected handlebody $N^{2 n}$ is determined completely by the rank $r$ of $H_{n}(M)$, the index $\tau$ of the intersection pairing, and the element $\chi^{2}=\langle\chi, \chi\rangle$. In the case at hand we have easily rank $H_{n}\left(N_{1}^{\prime}\right)=\operatorname{rank} H_{n}\left(N_{2}^{\prime}\right)$ and $\tau\left(N_{1}^{\prime}\right)=\tau\left(N_{2}^{\prime}\right)$. Finally, since $\chi\left(N_{1}^{\prime}\right)=\chi\left(N_{1}\right)+0$ and $\chi\left(N_{2}^{\prime}\right)=\chi\left(N_{2}\right)+\chi\left(N_{\lambda, j_{m}}\right)$ we have

$$
\begin{aligned}
\chi\left(N_{1}^{\prime}\right)^{2} & =2 \sum_{i=1}^{s} S \alpha_{1}\left(b_{i}\right) S \alpha_{1}\left(c_{i}\right) \\
& =2 \sum_{i=1}^{s}\left(S \alpha_{2}\left(b_{i}^{\prime}\right)+\beta_{i} j_{m}\right)\left(S \alpha_{2}\left(c_{i}^{\prime}\right)+\gamma_{i} j_{m}\right) \\
& =2 \sum_{i=1}^{s} S \alpha_{2}\left(b_{i}^{\prime}\right) S \alpha_{2}\left(c_{i}^{\prime}\right)+2 j_{m} \lambda \\
& =\chi\left(N_{2}\right)^{2}+\chi\left(N_{\lambda, j_{m}}\right)^{2} \\
& =\chi\left(N_{2}^{\prime}\right)^{2}
\end{aligned}
$$

since $\langle x, y\rangle=0$ for any elements $x \in H_{n}\left(N_{2}\right) \subset H_{n}\left(N_{2}^{\prime}\right)$ and $y \in H_{n}\left(N_{\lambda, j_{m}}\right) \subset H_{n}\left(N_{2}^{\prime}\right)$. Thus $N_{1}^{\prime}$ and $N_{2}^{\prime}$ determine the same element of $\mathscr{G}_{n}$. Since the map $N \rightarrow \partial N$ induces a homomorphism $\partial: \mathscr{G}_{n} \rightarrow \Theta_{2 n-1}$, it follows that $\partial N_{1}^{\prime}=\partial N_{2}^{\prime}$. Hence $\partial N_{1}=\partial N_{1}$ $\# S^{2 n-1}=\partial N_{1}^{\prime}=\partial N_{2}^{\prime}=\partial N_{2} \# \partial N_{\lambda, j_{m}}$ and Case 1 of the proposition follows.

Case 2. General case. Since $\hat{N}_{1}$ has the homotopy type of $\hat{N}_{2}$, the index $\tau\left(\hat{N}_{1}\right)=\tau\left(\hat{N}_{2}\right)$. But also 8 divides $\tau\left(\hat{N}_{k}\right), k=1,2$. Therefore there is a $\pi$-manifold $P$ with $\partial P \neq \varnothing$ such that $\tau(P)=\tau\left(\hat{N}_{k}\right)$. Consider the manifolds $M_{k}$ obtained by closing $N_{k}$ G $(-P), k=1,2$, where $-P$ denotes $P$ with the opposite orientation. Since $\hat{N}_{1}$ and $\hat{N}_{2}$ have the same homotopy type, a simple argument shows that $M_{1}$ and $M_{2}$ have the same homotopy type. But also $\tau\left(M_{k}\right)=\tau\left(\hat{N}_{k}\right)-\tau(P)=0, k=1,2$.

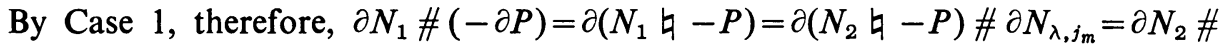
$-\partial P \# \partial N_{\lambda, i_{m}}$ for some $\lambda$. The lemma follows. 
Proof of 1.4. Under the identification $\mathscr{G}_{4 m}=Z \oplus Z \oplus Z$ of [12, Theorem 2, (1)], $N_{\lambda, \mu}$ corresponds to $(1,0, \lambda \mu)$. Since $\partial: \mathscr{G}_{4 m} \rightarrow \Theta_{4 m-1}$ depends only on the last two coordinates, the lemma follows.

\section{BIBLIOGRAPHY}

1. J. F. Adams, On the groups $J(X)$. IV, Topology 5 (1966), 21-71.

2. R. Bott, A note on the Samelson product in the classical groups, Comment. Math. Helv. 34 (1960), 249-256.

3. W. Browder, Homotopy type of differentiable manifolds, Colloquium on Algebraic Topology, Aarhus, 1962, pp. 42-46.

4. A. Haefliger, Plongements différentiables de variétés dans variétés, Comment. Math. Helv. 36 (1961), 47-82.

5. W. C. Hsiang, J. Levine and R. H. Szczarba, On the normal bundle of a homotopy sphere embedded in Euclidean space, Topology 3 (1965), 173-181.

6. M. Kervaire and J. W. Milnor, Bernoulli numbers, homotopy groups, and a theorem of Rohlin, Proc. Internat. Congr. Math., Edinburgh, 1958.

7. - Groups of homotopy spheres, $\Lambda \mathrm{nn}$. of Math. 77 (1963), 504-537.

8. A. Kosinski, On the inertia group of $\pi$-manifolds, Amer. J. Math. 89 (1967), 227-248.

9. M. Mahowald, On the order of the image of J, Topology 6 (1967), 371-378.

10. J. W. Milnor, Some differentiable structures on spheres, Amer. J. Math. 81 (1959), 962-972.

11. S. P. Novikov, Diffeomorphisms of simply connected manifolds, Dokl. Akad. Nauk SSSR 143 (1962), 1046-1049= Soviet Math. Dokl. 3 (1962), 540-543.

12. C. T. C. Wall, Classification of ( $n-1)$ connected $2 n$ manifolds, Ann. of Math. 75 (1962), 163-189.

NORTHWESTERN UNIVERSITY,

Evanston, ILLINOIS 